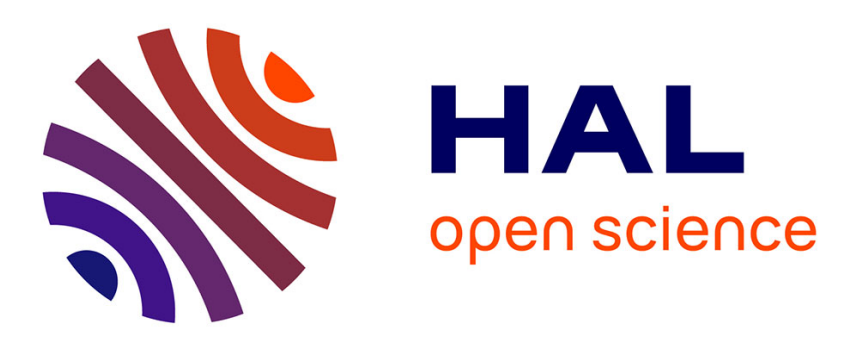

\title{
The singular power of the environment on stochastic nonlinear threshold Boolean automata networks
}

Jacques Demongeot, Sylvain Sené

\section{To cite this version:}

Jacques Demongeot, Sylvain Sené. The singular power of the environment on stochastic nonlinear threshold Boolean automata networks. 9th International Conference on Computational Methods in Systems Biology (CMSB 2011), Sep 2011, Paris, France. pp.55-64, 10.1145/2037509.2037518 . hal00666582

\section{HAL Id: hal-00666582 \\ https://hal.science/hal-00666582}

Submitted on 11 Feb 2014

HAL is a multi-disciplinary open access archive for the deposit and dissemination of scientific research documents, whether they are published or not. The documents may come from teaching and research institutions in France or abroad, or from public or private research centers.
L'archive ouverte pluridisciplinaire HAL, est destinée au dépôt et à la diffusion de documents scientifiques de niveau recherche, publiés ou non, émanant des établissements d'enseignement et de recherche français ou étrangers, des laboratoires publics ou privés. 


\title{
The singular power of the environment on stochastic nonlinear threshold Boolean automata networks
}

\author{
Jacques Demongeot ${ }^{1,3}$ and Sylvain Sené ${ }^{2,3}$ \\ 1 Université Joseph Fourier de Grenoble, AGIM, Pavillon Taillefer, Faculté de \\ Médecine, 38706 La Tronche cedex, France \\ ${ }^{2}$ Université d'Évry - Val d'Essonne, IBISC, 523 place des terrasses de l'agora, 91000 \\ Évry, France \\ 3 IXXI, Institut rhône-alpin des systèmes complexes, 5 rue du Vercors, 69007 Lyon,
}

France

\begin{abstract}
This paper tackles theoretically the question of the structural stability of biological regulation networks subjected to the influence of their environment. The model of networks considered is that of threshold Boolean automata networks that take place amongst the pertinent models for both neural and genetic regulation networks. Diving this study into the context of two-dimensional cellular automata on $\mathbb{Z}^{2}$ and modelling their environment by boundary conditions, this work analyses the dynamical behaviours of new kinds of threshold networks, namely stochastic nonlinear networks. Through an approach at the frontier between discrete dynamical system theory, probability theory and theoretical computer science combining formal and computer-assisted methods, we present under which specific characteristics of their parametric structure the dynamics of such networks in the attractive case is drastically subjected to the power of their environment.

Keywords: Nonlinear threshold Boolean automata networks, stochastic processes, structural stability, environment, biological regulation networks
\end{abstract}

\section{Introduction}

Threshold Boolean automata networks (TBANs for short) constitute a "simple" mathematical model of discrete dynamical systems at the centre of numerous studies. They were introduced in the 1940's by McCulloch and Pitts [1] in order to provide a logical representation of neurons activities. It is in the 1980's that major studies, from the domains of mathematics and physics, focused on its formal properties. In particular, Goles and Hopfield, respectively in $[2,3,4]$ and $[5,6,7]$, presented results highlighting strong dynamical and computational properties of such networks under specific constraints (such as symmetry), like global characterisations of their dynamics. At the same time, studies introducing methods for modelling genetic regulation networks had already been published by Kauffman and Glass $[8,9]$, and Thomas $[10,11]$. Since then, discrete bio-mathematics and 
bio-informatics have been widely developed and have led to use TBANs also as models of genetic regulation networks $[12,13]$.

It is in this large context, at the frontier between mathematics, physics and theoretical biology, that the present work takes place. In these domains, amongst the questions that are nowadays central is that of systems robustness, defined here as structural stability, that is the ability of systems to keep their asymptotic behaviours when they are subjected to perturbations of structural parameters [14]. Amongst the robustness types, numerous studies have considered the environments as a crucial notion, in particular in the context of neural networks $[15,16]$ as well as in that of gene regulation networks $[17,18]$. Here, we propose to present new elements showing that the question of how can the environment of a system influence the latter is all the more pertinent in the theoretical framework of stochastic nonlinear TBANs (abbreviated by SNTBANs for the sake of simplicity).

More precisely, our purpose is to understand to what extent different boundary conditions can lead such networks to behave profoundly differently. This is enabled thanks to the proposition of a theoretical measure of these fundamental behavioural differences (i.e., a phase transitions measure) that is translatable into simulations. Although the subject of this work is fairly that of the study of the dynamics emerging from the couple SNTBANs/environment, it is important to note that it is also close to studies performed on ferromagnetism in physics. Indeed, at the end of the 1960's, studies addressed the problem of phase transitions in the classical Ising model [19], from different points of view [20,21,22]. Other studies focused on nonlinear Ising-like models and showed that the introduction of triplet potentials led to new kinds of phase transitions [23,24,25]. Basing ourselves on recent studies we performed on linear TBANs [26,27], we present new results leaned on discrete dynamical system theory and simulations. Our contribution consists in obtaining new results that generalise to the case of general nonlinear networks (i.e., networks taking into account any kind of neighbourhood coalition interaction potential, namely triplet as well as quadruplet, quintuplet and even sextuplet potentials) well known results on the classical Ising model $[21,22]$ and other results we obtained in the restricted case of linear TBANs $[26,27,28]$. Precisely, we present a computer-assisted approach yielding an empirical condition of emergence of phase transitions, which leads to provide a fine analysis of the singular power of the environment on SNTBANs. Note that the results presented in this paper cannot be directly related to real biological networks such as genetic ones because of the purely theoretical nature of networks considered. Nevertheless, the main objective of this paper is twofold: (i) provide a supplementary insight (in addition to results given in $[26,28]$ ) that studying properly the dynamical behaviours of networks opened to their environment implies to understand under which conditions such an environment can be impacting ${ }^{4}$, and (ii) show that the concept of nonlinearity addressed makes sense

\footnotetext{
4 Beyond the fact that our argumentation focuses here on very constrained networks, we will discuss at the end of this paper that this insight has already been verified on real biological networks.
} 


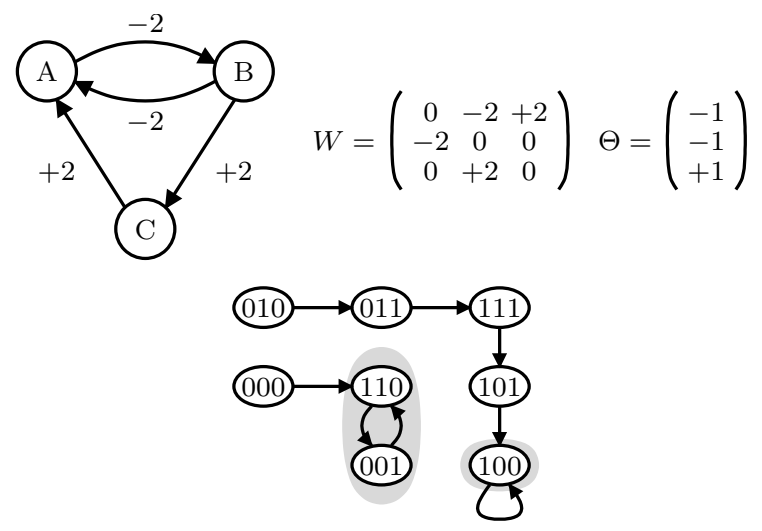

Fig. 1. (Top) A tBAn composed of $\mathbf{3}$ automata with its interaction graph, its interaction matrix $\mathbf{W}$ and its threshold vector $\boldsymbol{\Theta}$; (Bottom) its associated transition graph depending on the parallel iteration $(\{\mathbf{A}, \mathbf{B}, \mathbf{C}\})$ that shows the existence of two attractors, a fixed point (100) and a limit cycle of period 2 $(110 \rightleftarrows 001)$.

in modelling real biological networks and could be useful in order to represent protein complexes for instance.

After we present in Section 2 important notions of which we make specific use throughout the paper, Section 3 provides a description of a dissimilarity measure of the dynamical behaviours of SNTBANs implemented in a Monte-Carlo algorithm for simulations. Then, we propose in Section 4 a formal analysis of the problem of emergence of phase transitions leading to the emphasis of theoretical results highlighting conditions of influence of the environment ensued by simulation results. Eventually, we discuss some perspectives of this work and insist on biological applications that could be derived from the notions of nonlinearity and boundary conditions.

\section{Preliminaries}

\subsection{Classical TBANs definition}

Although this work focuses on two-dimensional finite SNTBANs dived into the regular lattice $\mathbb{Z}^{2}$, let us begin by defining classical deterministic TBANs, called simply TBANs, from the general point of view [1,29]. An arbitrary TBAN $N$ of size $n$ is composed of $n$ automata interacting with each others over discrete time (time space $\mathcal{T}$ is a subset of $\mathbb{N}$ ) through the links they share, classically called interactions or regulations. Any of the automata $i \in\{1, \ldots, n\}$ of $N$ owns a state $x_{i}$ valued in $\{0,1\}$ (0 means that $i$ is inactivated/inhibited and 1 that $i$ is activated/expressed). Considering that $x_{i}(t)$ is the local state of automaton $i$ at time $t$, we derive the notion of global state at time $t$, called configuration in the sequel for the sake of clarity, that is a vector $x(t)=\left(x_{i}(t)\right)_{i \in\{1, \ldots, n\}} \in\{0,1\}^{n}$. 
Definition 1. A TBAN $N$ of size $n$ (i.e., composed of $n$ automata) is a triplet $(W, \Theta, F)$ where:

- $W$ is an interaction matrix of order $n$, where coefficient $w_{i, j} \in \mathbb{R}$ represents the interaction weight that automaton $j$ has on automaton $i$;

- $\Theta$ is an activation threshold vector of dimension $n$, in which $\theta_{i}$ is the activation threshold attributed to automaton $i$;

- $F:\{0,1\}^{n} \rightarrow\{0,1\}^{n}$, such that $F=\left(f_{1}, \ldots, f_{n}\right)$, is a global transition function, i.e., a vector of $n$ local transition functions in which function $f_{i}:\{0,1\}^{n} \rightarrow\{0,1\}$ computes the new state of automaton $i$ at time $t+1$ according to $W, \Theta$ and the configuration of $N$ at time $t$ such that:

$$
f_{i}(x)=x_{i}(t+1)= \begin{cases}1 & \text { if } \sum_{j \in \mathcal{N}_{i}} w_{i, j} \cdot x_{j}(t)-\theta_{i}>0 \\ 0 & \text { otherwise }\end{cases}
$$

where $\mathcal{N}_{i}$ is the neighbourhood of automaton $i$, i.e., the set of automata $j$ such that $w_{i, j} \neq 0$.

From now, $N$ represents an arbitrary deterministic TBAN of size $n$. Its interaction matrix $W$ characterises the structure of $N$ and is the algebraic representation of a labelled digraph $G=(V, E)$, where $V=\{1, \ldots, n\}$ is a set of vertices (i.e., the automata of $N)$ and $E \subseteq V \times \mathbb{R}^{*} \times V$ is the set of labelled arcs linking automata with each others that represent the directed interactions between them. $G$ is classically called the interaction graph associated with $N$. Labels $w_{i, j}$ 's are called interaction weights. Note that when a coefficient $w_{i, j}$ of $W$ is null, automaton $j$ has no influence on automaton $i$ and there is consequently no arc from $j$ to $i$ in $G$. In terms of genetic regulation networks, that means that the protein produced by the expression of gene $j$ does not take part in the regulation process of gene $i$. Conversely, if $w_{i, j} \neq 0, j$ tends to influence $i$ and there is an arc from $j$ to $i$ in $G$. From the genetic point of view, if $w_{i, j}>0$ (resp. if $\left.w_{i, j}<0\right)$ then gene $j$, when expressed, impacts positively (resp. negatively) the expression of gene $i$, which means that $j$ is an activator (resp. an inhibitor) of $i$. If automata represent neurons, interaction weights are classically called synaptic weights and $w_{i, j}$ represents the electric potential of firing that $j$ has on $i$. Of course, the bigger $\left|w_{i, j}\right|$ is, the more important the (positive or negative) influence of $j$ on $i$ is. An illustration of a TBAN is presented in Figure 1 (Top panel).

\section{$2.2 \quad$ Updating schedule}

TBANs are deterministic discrete dynamical systems, in the sense that the image at time $t+1$ of a configuration $x$ at time $t$ is unique and determined by the deterministic global transition function $F$ with which is associated a specific discrete iteration. Numerous discrete iterations can be defined. In this work, we chose to focus on the particular parallel discrete iteration (parallel iteration for short). 


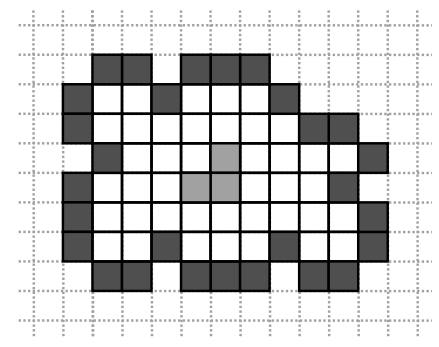

Fig. 2. Architecture of a tBAN $\mathbf{N}$ on $\mathbb{Z}^{\mathbf{2}}$. Automata of $\mathbf{N}$ are represented by white and light grey (in the case of central nodes of eccentricity 7 ) square cells. Boundary $\partial_{\text {ext }} \mathbf{N}$ is the set of cells coloured in dark grey.

Definition 2. Given a TBAN $N$ whose interaction graph is $G=(V, E)$, the parallel iteration on $N$, denoted by the ordered partition $(\{1, \ldots, n\})$, is defined as:

$$
\forall t \in \mathcal{T}, \forall x=x(t) \in\{0,1\}^{n}: F(x)=x(t+1)=\left(f_{1}(x), f_{2}(x), \ldots, f_{n}(x)\right) .
$$

As far as we are concerned, this choice does not reduce the range of our results. Indeed, previous results $[26,28]$ show that if the influence of the environment is effective with the parallel iteration, it is also effective with most of the other discrete iterations defined as Robert's according to ordered partitions of $V$ [30,31]. Furthermore, the dynamics of $N$ resulting from the parallel iteration can be represented by a transition graph $\mathcal{G}=\left(\{0,1\}^{n}, T r\right)$, where $\operatorname{Tr}=\left\{(x(t), x(t+1)) \mid x(t), x(t+1) \in\{0,1\}^{n}\right\}$ denotes the possible transitions between configurations of $N$. Thus, $\mathcal{G}$ pictures the trajectories from all possible configurations to the limit sets of the successive applications of $F$. Since $\{0,1\}^{n}$ is finite, the limit set of $F$ is restricted to two kinds of attractors, (i) fixed points that are configurations $x$ such that $x(t+1)=x(t)$ and (ii) limit cycles that are circuits of configurations in $\mathcal{G}$, i.e., sets of configurations that repeat themselves endlessly (see Figure 1 (Bottom panel)).

\subsection{Boundary, centre and specific restrictions}

We give now more details about structural elements of the TBANs considered. From now, $N$ denotes a TBAN whose underlying interaction graph $G=(V, E)$ is dived into $\mathbb{Z}^{2}$, in which the neighbourhood $\mathcal{N}_{i}$ of each of the elements $i \in V$ is the set composed of itself and its nearest automata, i.e., automata of $\mathbb{Z}^{2}$ located at distance not greater than 1 to $i$ in terms of Manhattan distance. Note that the previous sentence does not replace the definition of neighbourhood given above in the context of general TBANs (not restricted to $\mathbb{Z}^{2}$ ) and consists only in a topological precision of this notion in $\mathbb{Z}^{2}$. Indeed, considering an automaton $i \in V$ and denoting by $V^{c}=\mathbb{Z}^{2} \backslash V$ the set of automata of $N^{c}$ (said to be the complement of $N$ in $\mathbb{Z}^{2}$ ), then the neighbourhood of $i$, i.e., the set of automata 
that influence $i$ such that $w_{i, j} \neq 0$, is $\left\{j \in V \cup V^{c} \mid d_{M}(i, j) \leq 1\right\}$, where $d_{M}(i, j)$ denotes the Manhattan distance between $i$ and $j$. Let us now add two definitions about the notions of isotropy and translation invariance. To do so, we first denote by $\Lambda_{i}=\mathcal{N}_{i} \backslash\{i\}$ the strict neighbourhood of automaton $i \in V$. Then, a TBAN $N$ on $\mathbb{Z}^{2}$ is said to be isotropic if and only if $\forall i \in V, \forall j, j^{\prime} \in$ $\Lambda_{i}: w_{i, j}=w_{i, j^{\prime}}$. A TBAN $N$ on $\mathbb{Z}^{2}$ is translation invariant if and only if $\forall i, i^{\prime} \in$ $V, s=i^{\prime}-i, \forall j \in \Lambda_{i}: w_{i, j}=w_{i^{\prime}, j+s}$. In this paper, TBANs considered are finite, bounded, isotropic and translation invariant, which implies that they are symmetric, i.e., $\forall i, j \in V: w_{i, j}=w_{j, i}$. As a consequence, all local transition functions are identical. Hence, TBANs studied are cellular automata. Moreover, they are attractive, i.e., such that $\forall i \in V, \forall j \in \Lambda_{i}:\left(w_{i, i} \leq 0\right) \wedge\left(w_{i, j} \geq 0\right)$. From this, we derive the definitions of two central notions that we will make specific use in the sequel, that of centre and that of boundary. Let the digraph metric $d(u, v)$ between $u$ and $v$, where $u, v \in V$, be defined as the length of the shortest path from $u$ to $v$. Note that if such a path does not exist then $d(u, v)=\infty$. The eccentricity $\varepsilon(u)$ of $u$ is the maximal digraph metric from $u$ to every other vertex of $G$ such that $\varepsilon(u)=\operatorname{Max}_{v \in V \backslash\{u\}}[d(u, v) \neq \infty]$.

Definition 3. The centre of a TBAN $N$ associated with an interaction graph $G=(V, E)$ is the set $V^{\prime} \subseteq V$ whose elements are vertices of minimal eccentricity.

Boundaries are built as in [32] (see Figure 2).

Definition 4. The boundary of a TBAN $N$ on $\mathbb{Z}^{2}$, denoted by $\partial_{\text {ext }} N$, is the subset of nodes of $V^{c}$ such that:

$$
\partial_{\text {ext }} N=\left\{i \in V^{c} \mid \exists j \in V: i \in \mathcal{N}_{j}, j \notin \mathcal{N}_{i}\right\} .
$$

A boundary condition is then simply defined as the allocation of a state value to each node of $\partial_{\text {ext }} N$.

\subsection{Stochastic nonlinear networks}

Let us denote by $N^{*}$ the TBAN composed of both automata of $N$ and boundary automata of $\partial_{\text {ext }} N$, such that its associated interaction graph is $G^{*}=$ $\left(V^{*}, E^{*}\right)$ where $V^{*}=V \cup \partial_{\text {ext }} V$ and $E^{*}=E \cup\left\{\left(i, w_{j, i}, j\right) \mid i \in \partial_{\text {ext }} V, j \in V\right\}$. We also make a specific use of the notion of interaction potentials for each $i$ belonging to $N$, introducing a parameter $T \in \mathbb{R}^{+}$, classically called the temperature parameter. The singleton potential is $u_{0, i}=\frac{w_{i, i}}{T}$, the couple potential is $\forall j \in \Lambda_{i}: u_{1, i, j}=\frac{w_{i, j}}{T}$, the triplet potential is $\forall j, \ell \in \mathcal{N}_{i}$ s.t. $j \neq \ell: u_{2, i,\langle j, \ell\rangle}=$ $\frac{w_{i,\langle j, \ell\rangle}}{T}, \ldots$, the sextuplet potential is $\forall i, j, \ell, m, p \in \mathcal{N}_{i}$ s.t. $i \neq j \neq \ell \neq m \neq$ $p: \quad u_{5, i,\langle i, j, \ell, m, p\rangle}=\frac{w_{i,\langle i, j, \ell, m, p\rangle}}{T}$. For the sake of clarity, note that, for instance, the triplet potential $u_{2, i,\langle j, \ell\rangle}$ is the specific interaction weight normalised by $T$ that the set of the two distinct active automata $j$ and $\ell$ of $\mathcal{N}_{i}$ have together on $i$. In other words, it denotes the interaction potential that the group composed of $j$ and $\ell$ together and seen as a new kind of interacting entity has on $i$. Figure 3 pictures, for an arbitrary automaton $i$, its possible neighbourhood configurations 


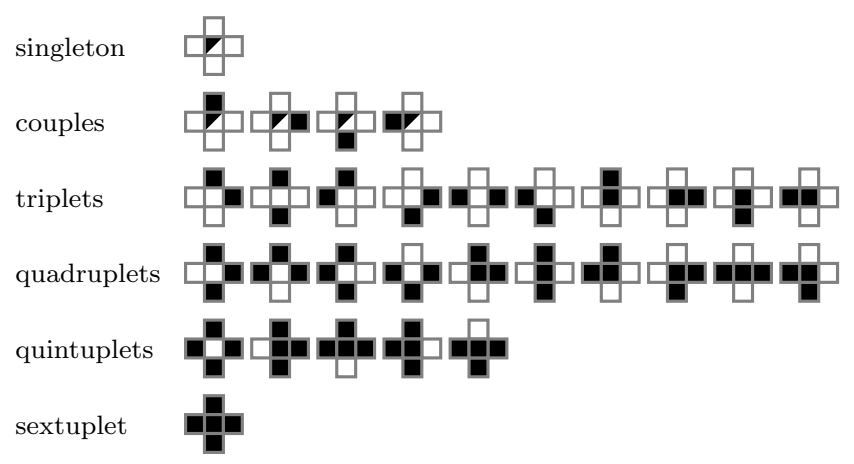

Fig. 3. Representation of the possible configurations in the neighbourhood of an arbitrary automaton $\mathbf{i}$ of a TBAN whose automata are vertices of in $\mathbb{Z}^{2}$. Black (resp. white) cells represent active (resp. inactive) nodes. In the two first lines, the central automaton is both black and white, which means that its state has not to be considered in the interaction potentials.

ordered according to the possible interaction potentials $i$ has to account when computing its new state. Note that the interaction potentials are "cumulative" in the sense that a configuration inducing to account a triplet potential induces also to account from 1 to 2 couple potentials, depending on the fact that $i$ belongs or not to the group acting on itself, and 1 singleton potential (which always takes part in the computation of a new state). Thus, interaction potentials lead us to provide the following definition.

Definition 5. Let $G=(V, E)$ a digraph whose vertices are automata in $\mathbb{Z}^{2}$. A two-dimensional stochastic TBAN of size $n$ and order $k$, with $(2 \leq k \leq 6)$, on $\mathbb{Z}^{2}$ associated with $G$ is a TBAN whose local transition functions are stochastic and defined by the following probabilistic function by accounting exclusively 1-tuple, $\ldots, k$-tuple potentials terms:

$$
\begin{aligned}
& \forall i \in V=\{1, \ldots, n\}: \\
& \qquad P\left(x_{i}(t+1)=\alpha\right)=\frac{e^{\alpha \cdot\left(u_{0, i}+\sum_{j \in \Lambda_{i}} u_{1, i, j} \cdot x_{j}(t)+\eta_{i}^{k}\left(\Lambda_{i}\right)\right)}}{1+e^{u_{0, i}+\sum_{j \in \Lambda_{i}} u_{1, i, j} \cdot x_{j}(t)+\eta_{i}^{k}\left(\Lambda_{i}\right)}},
\end{aligned}
$$

where $\eta_{i}^{k}\left(\Lambda_{i}\right)$ is a nonlinear interaction potential (also called nonlinear term) such that:

$$
\eta_{i}^{k}\left(\Lambda_{i}\right)= \begin{cases}0 & \text { if } k=2, \\
\sum_{\substack{j_{1}, j_{2} \in \mathcal{N}_{i} \\
j_{1} \neq j_{2}}} u_{2, i,\left\langle j_{1}, j_{2}\right\rangle} \cdot x_{j_{1}}(t) \cdot x_{j_{2}}(t) & \text { if } k=3, \\
\begin{array}{c}
\sum_{j_{1}, \ldots, j_{k-1} \in \mathcal{N}_{i}} u_{2, i,\left\langle j_{1}, j_{2}\right\rangle} \cdot x_{j_{1}}(t) \cdot x_{j_{2}}(t)+\ldots+\ldots \neq j_{k-1} \\
j_{1} \neq \ldots \neq \ldots
\end{array} & \\
u_{k-1, i,\left\langle j_{1}, \ldots, j_{k-1}\right\rangle} \cdot x_{j_{1}}(t) \cdot x_{j_{2}}(t) \cdot \ldots \cdot x_{j_{k-1}}(t) & \text { otherwise. }\end{cases}
$$

From Definition 5, it follows that stochastic TBANs of order $k=2$ are stochastic versions of classical TBANs and that SNTBANs are stochastic TBANs of order 
$k \geq 3$. Hence, SNTBAns are Boltzmann machines [33,34] extended to account several kinds of nonlinear interaction potentials. More precisely, all local transition functions compute probabilities of nodes to be at state $\alpha \in\{0,1\}$ at time $t+1$ according to their neighbourhood configurations at time $t$. It follows directly that, for any $t \in \mathcal{T}$, depending on a global configuration $x$ at time $t$, the global transition function $F$ computes the probability for $x$ to become any other configuration $x^{\prime} \in\{0,1\}^{n}$ at time $t+1$. Consequently, it derives that the dynamics of SNTBANs of size $n$ are stationary Markov chains whose random variables are the $2^{n}$ possible configurations such that:

$$
\forall t \in \mathbb{N}^{*}: P(x(t+1) \mid x(t))=P(x(t) \mid x(t-1)) .
$$

\section{Dissimilarity measure}

In $[20,21]$, Dobrushin characterised phase transitions in the Ising model as domains of structural parameters under which the underlying Markov chain was not ergodic anymore.

Definition 6. Let $C$ be the stationary Markov chain associated with the dynamics of a SNTBAN $N$. The Markovian matrix $\mathcal{P}$ of $C$ is a matrix of order $2^{n}$ whose coefficients are such that:

$$
\forall i, j \in\{0,1\}^{n}: p_{i, j}=P(x(t+1)=j \mid x(t)=i) .
$$

Definition 7. Let $C$ be the stationary Markov chain associated with the dynamics of a SNTBAN $N$ and $\mathcal{P}$ its underlying Markovian matrix. The invariant measure of $C$ is the vector $\mu$ whose entries are non-negative and sum to 1 that satisfies:

$$
\mu_{j}=\sum_{i \in\{0,1\}^{n}} \mu_{i} p_{i, j} .
$$

In other words, an invariant measure $\mu$ is a normalised left eigenvector of the Markovian matrix associated with the eigenvalue 1 which constitutes the matching attractor in the framework of stochastic dynamical systems ${ }^{5}$. Thus, basing our work in the framework of stochastic processes and ergodic theory, with a method close to that of Dobrushin, we analyse the influence of boundary conditions on SNTBANs by showing under which structural parameters networks are subjected to phase transitions. To do so, we make specific use of the notion of invariant measures.

Definition 8. Let $N$ be an arbitrary SNTBAN, $\partial_{\mathrm{ext}}^{0} N$ and $\partial_{\mathrm{ext}}^{1} N$ be two different boundary conditions of $N$ and let $\mu_{0}$ (resp. $\mu_{1}$ ) be the invariant measure associated with the Markov chain defining the evolution of $N^{0}=N \cup \partial_{\mathrm{ext}}^{0} N$ (resp. $\left.N^{1}=N \cup \partial_{\mathrm{ext}}^{1} N\right)$. A phase transition is said to emerge from the dynamical behaviour of $N$ if and only if $\mu_{0} \neq \mu_{1}$ when $n$ tends to infinity.

\footnotetext{
5 Markovian matrices we are interested in contain only positive coefficients. Then, the PerronFrobenius theorem can be applied, which proves the uniqueness of the invariant measure for any system $N^{*}$.
} 
We will say that $N$ depends on its environment when a phase transition emerges from its asymptotic dynamical behaviour. Now, let us explain the method chosen to obtain a computational representation of this notion of invariant measure. An invariant measure gives the occurrence frequency of each of the configurations of $N$ when the SNTBAN evolves over time, when time tends to infinity. The evolution of such a network comprises two periods: (i) the transient period during which the empirical frequencies of the global network states, calculated from time $t=0$, have not already converged to the invariant measure frequencies, and (ii) the stable period, during which the invariant measure gives precisely the occurrence frequency of every configuration asymptotically. Hence, from the local point of view of an automaton $i$, its activity (i.e., the number of asymptotic iterations during which node $i$ has been active) can be derived from the invariant measure. Consequently, we define the dissimilarity measure, which gives a value to the influence of boundary conditions, from the asymptotic activity of one automaton $O$ belonging to the centre of a SNTBAN, which is a priori the less impacted by boundary conditions because of its position on the lattice. From now, $O$ refers to the central automaton chosen for the study. Let $T_{t}$ be the transient time during which the system evolves to reach its asymptotic (stable) behaviour and $T_{s}$ be the sampling time, occurring after $T_{t}$, during which the (stable) activity $\mathcal{A}$ of $O$ is recorded. We write: $\mathcal{A}=\sum_{t \in T_{s}} x_{O}(t)$. Considering now the two networks $N^{0}$ and $N^{1}$ described above, we compute the corresponding central activities $\mathcal{A}^{0}$ and $\mathcal{A}^{1}$ and define the dissimilarity measure $S$ as:

$$
S=\frac{\left|\mathcal{A}^{0}-\mathcal{A}^{1}\right|}{\left|T_{s}\right|}
$$

Now the context is clearly identified, we are going to present the computerassisted approach providing an empirical condition for the environment to be impacting the dynamical behaviour of attractive SNTBANs.

\section{Computer - assisted analysis of structural instability}

\subsection{Theoretical approach}

From now, $N$ denotes an arbitrary attractive SNTBAN on $\mathbb{Z}^{2}$ of size $n$ and order $k(3 \leq k \leq 6)$ with $G=(V, E)$ its underlying interaction graph, $C$ its associated Markov chain whose related Markovian matrix is $\mathcal{P}$. Using set theory terminology, let us first redefine a configuration by introducing the concept of cylinder.

Definition 9. In a SNTBAN $N$, a cylinder $[A, B]$ is a set composed of one configuration $x \in\{0,1\}^{n}$ such that:

$$
\forall A, B \subseteq V \text { s.t. } A \cap B=\emptyset:[A, B]=\left\{x \mid \forall i \in A: \quad x_{i}=1 ; \forall i \in B: x_{i}=0\right\} .
$$


If $\mu$ denotes the invariant measure of $C$ when the size of $N$ tends to infinity, by definition, $\mu$ satisfies the following projectivity and conditional relations. Projectivity equations are defined as:

$$
\begin{aligned}
\forall A, B \subseteq V \text { s.t. } A \cap B=\emptyset, \forall i \in A: & \\
& \mu([A, B])+\mu([A \backslash\{i\}, B \cup\{i\}])=\mu([A \backslash\{i\}, B]),
\end{aligned}
$$

where $\mu([A, B])$ is the asymptotic probability to observe the configuration represented by $[A, B]$. Conditional equations (i.e., Bayes formulae), are then defined as:

$$
\forall i \in V, \forall A, B \subseteq V \text { s.t. } A \cap B=\emptyset: \mu([\{i\}, \emptyset])=\sum_{A, B} \Phi_{i}(A, B) \cdot \mu([A, B]),
$$

where $\mu([\{i\}, \emptyset])$ represents the asymptotic global probability that automaton $i$ is at state 1 and $\Phi_{i}(A, B)$ denotes the conditional probability given in Equation 1 that the state of $i$ at time $t+1$ equals 1 knowing $[A, B]$ at time $t$ such that:

$$
\mu\left(x_{i}(t+1)=1 \mid[A, B]\right)=\Phi_{i}(A, B)=\frac{e^{u_{0, i}+\sum_{j \in \Lambda_{i} \cap A} u_{1, i, j}(t)+\eta_{i}^{k}\left(\Lambda_{i}\right)}}{1+e^{u_{0, i}+\sum_{j \in \Lambda_{i} \cap A} u_{1, i, j}(t)+\eta_{i}^{k}\left(\Lambda_{i}\right)}},
$$

where, denoting $\left(\Lambda_{i} \cap A\right) \cup(\{i \mid i \in A\})$ by $\Xi_{i}$ :

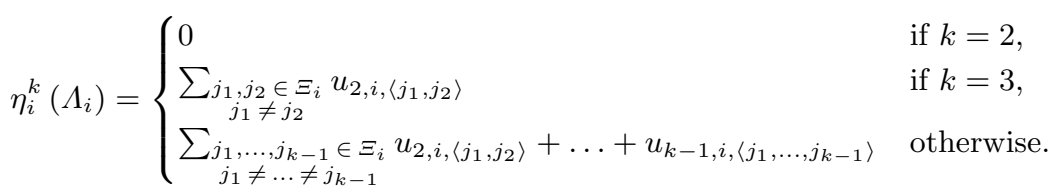

Because of the translation invariant character of networks studied, any SNTBAN owns a spatial Markovian character that allows the study of the dynamical behaviour of an arbitrary SNTBAN $N^{\prime}$ whose size tends to infinity to be reduced to the SNTBAN $N$ whose underlying interaction graph $G$ is the subgraph of $G^{\prime}$ restricted to vertices in the neighbourhood $\mathcal{N}_{O^{\prime}}=\mathcal{N}_{O}$ of the centre $O^{\prime}$ of $N^{\prime}$. Consider the strict neighbourhood $\Lambda_{O}=\mathcal{N}_{O} \backslash\{O\}=\{1,2,3,4\}$ of the central automaton $O$. Thence, we introduce a matching Markovian matrix $\mathcal{P}$ with the concept of projectivity matrix ${ }^{6}$.

Definition 10. Let $N$ be a stochastic TBAN of size $n$ and order $k$ on $\mathbb{Z}^{2}$. The projectivity matrix $\mathcal{M}$ of $N$ is a matrix of order $2^{\left|\Lambda_{O}\right|}$ whose coefficients are those of the following system of linear projectivity and conditional equations (see

\footnotetext{
${ }^{6}$ The general definition of the projectivity matrix $\mathcal{M}$ of arbitrary general TBANs of order 2 dived into $\mathbb{Z}^{d}$ is given in [28].
} 
Equations 2 and 3) in which unknowns are the $\mu$ 's:

$$
\left\{\begin{array}{l}
\mu([\{1,2,3,4\}, \emptyset])+\mu([\{2,3,4\},\{1\}])=\mu([\{2,3,4\}, \emptyset]) \\
\mu([\{1,2,3,4\}, \emptyset])+\mu([\{1,3,4\},\{2\}])=\mu([\{1,3,4\}, \emptyset]) \\
\mu([\{1,2,3,4\}, \emptyset])+\mu([\{1,2,4\},\{3\}])=\mu([\{1,2,4\}, \emptyset]) \\
\mu([\{1,2,3,4\}, \emptyset])+\mu([\{1,2,3\},\{4\}])=\mu([\{1,2,3\}, \emptyset]) \\
\mu([\{2,3,4\},\{1\}])+\mu([\{3,4\},\{1,2\}])=\mu([\{3,4\},\{1\}]) \\
\mu([\{2,3,4\},\{1\}])+\mu([\{2,4\},\{1,3\}])=\mu([\{2,4\},\{1\}]) \\
\mu([\{2,3,4\},\{1\}])+\mu([\{2,3\},\{1,4\}])=\mu([\{2,3\},\{1\}]) \\
\mu([\{1,3,4\},\{2\}])+\mu([\{1,4\},\{2,3\}])=\mu([\{1,4\},\{2\}]) \\
\mu([\{1,3,4\},\{2\}])+\mu([\{1,3\},\{2,4\}])=\mu([\{1,3\},\{2\}]) \\
\mu([\{1,2,4\},\{3\}])+\mu([\{1,2\},\{3,4\}])=\mu([\{1,2\},\{3\}]) \\
\mu([\{3,4\},\{1,2\}])+\mu([\{4\},\{1,2,3\}])=\mu([\{4\},\{1,2\}]) \\
\mu([\{3,4\},\{1,2\}])+\mu([\{3\},\{1,2,4\}])=\mu([\{3\},\{1,2\}]) \\
\mu([\{2,4\},\{1,3\}])+\mu([\{2\},\{1,3,4\}])=\mu([\{2\},\{1,3\}]) \\
\mu([\{1,4\},\{2,3\}])+\mu([\{1\},\{2,3,4\}])=\mu([\{1\},\{2,3\}]) \\
\mu([\{4\},\{1,2,3\}])+\mu([\emptyset,\{1,2,3,4\}])=\mu([\emptyset,\{1,2,3\}]) \\
\sum_{A, B \subseteq A} \Phi_{O}(A, B) \cdot \mu([A, B]) \\
A \cap B=\emptyset
\end{array}\right.
$$

Projectivity and conditional equations of the linear system of Definition 10 are in general linearly independent. In $[26,27]$, we have shown that the linear dependency of these equations was a necessary condition for attractive classical stochastic TBANs to be subjected to phase transitions. This result leads us to formulate the following claim.

Claim. If attractive SNTBANs are structurally unstable (i.e., non-robust) against fluctuations of their environment, then the determinant of their underlying projectivity matrix is null.

Clearly, this claim is based on the fact that phase transitions generally only occur when structural parameters that characterise systems are intimately related $[20,22]$. In the system of linear equations given above, this corresponds to a linear dependency between entries. Thus, considering the claim above as the central hypothesis of our theoretical reasoning, we are now going to find a formal sufficient condition on SNTBANs that validates the nullity of the determinant of their projectivity matrices. We will emphasise in Section 4.3 by simulations that this condition is the only one for which the environment of attractive SNTBANs has a significant impact on their dynamical behaviours.

\subsection{Theoretical results}

In 1981, Demongeot analysed some properties of Markov random fields and obtained a general formula characterising the nullity of the determinant of projectivity matrices [35] such as those described above. Dived into our framework, that resulted in the following lemma of which we will make a specific use. 
Lemma 1. [35] The nullity of the determinant of a projectivity matrix $\mathcal{M}$ is characterised by:

$$
\operatorname{Det} \mathcal{M}=0 \Longleftrightarrow \sum_{K \subseteq \Lambda_{O}}(-1)^{\left|\Lambda_{O} \backslash K\right|} \cdot \Phi_{O}\left(K, \Lambda_{O} \backslash K\right)=0 .
$$

As we have evoked, our aim is now to highlight formally a specific sufficient condition on an arbitrary attractive SNTBAN $N$, whose asymptotic dynamics is described by a projectivity matrix $\mathcal{M}$, that implies the nullity of Det $\mathcal{M}$.

Definition 11. Let $i$ be an arbitrary automaton $i$ of a SNTBAN $N$. The nonlinear interaction potential of $i$, denoted by $\eta_{i}^{k}\left(\Lambda_{i}\right)$, is symmetric if and only if:

$$
\forall K \subseteq \Lambda_{i}: \eta_{i}^{k}\left(\Lambda_{i}\right)=\eta_{i}^{k}(K)+\eta_{i}^{k}\left(\Lambda_{i} \backslash K\right) .
$$

The result that will be given emphasises that the symmetry of the nonlinear term of Equation 1 is sufficient for Det $\mathcal{M}=0$ to hold. Note that the choice of this specific property for the nonlinear term comes directly from the claim above. According to Claim 4.1, Det $\mathcal{M}=0$ is a necessary condition for phase transitions to emerge. This condition on Det $\mathcal{M}$ means that there exists a specific relation between at least two equations of the linear system presented in Definition 10. By extension, that means that there exists a specific relation between the interaction potentials $u$ that define SNTBANs. Our past studies on linear TBANs $[26,28,36]$ have shown that this peculiar relation is a counter-balancing relation between negative singleton potentials and positive couple potentials. From this knowledge, it seemed natural that the same kind of counter-balancing relation occurs in SNTBANs. Now, it suffices to remark that the symmetry of the non-linear term constitutes a way to build non-linear potentials of different parities of different signs in order to favour the counter-balancing effect.

Thereby, in the sequel, let us consider that, for any $K \subseteq \Lambda_{O}$, the nonlinear term $\eta_{O}^{k}(K)$ is symmetric and equals $-2 \cdot u_{0, O}-\sum_{j \in \Lambda_{O}} u_{1, O, j}-\eta_{O}^{k}\left(\Lambda_{O} \backslash K\right)$. The specific conditions of nonlinearity precised in Definition 5 and the other conditions of isotropy, translation invariance and attractiveness that networks studied satisfy lead us to emphasise necessary and sufficient conditions that parameters $u$ 's must respect. First, Lemma 2 gives a characterisation of the symmetric nonlinear term.

Lemma 2. Let $N$ be an arbitrary SNTBAN of order $k$. Given an arbitrary $K \subseteq$ $\Lambda_{O}$ and the nonlinear term on $K$ defined by $\eta_{O}^{k}(K)=-2 \cdot u_{0, O}-\sum_{j \in \Lambda_{O}} u_{1, O, j}-$ $\eta_{O}^{k}\left(\Lambda_{O} \backslash K\right)$, the symmetry property of the nonlinear interaction potential of $N$ verifies:

$$
\begin{aligned}
\forall K \subseteq \Lambda_{O}: \eta_{O}^{k}(K)=\eta_{O}^{k}\left(\Lambda_{O}\right)-\eta_{O}^{k}\left(\Lambda_{O} \backslash K\right) \Longleftrightarrow \\
u_{0, O}+\frac{\sum_{j \in \Lambda_{O}} u_{1, O, j}}{2}+\frac{\eta_{O}^{k}\left(\Lambda_{O}\right)}{2}=0 .
\end{aligned}
$$


Proof. Let set $K$ be defined as $K \subseteq \Lambda_{O}$. Denoting $\eta_{O}^{k}\left(\Lambda_{O}\right)-\eta_{O}^{k}\left(\Lambda_{O} \backslash K\right)=\eta_{\text {sym }}$ and developing the left member of Equation 4 by definition of nonlinear terms, trivially, we have:

$$
\begin{aligned}
\eta_{O}^{k}(K)=\eta_{\mathrm{sym}} & \Longleftrightarrow-2 \cdot u_{0, O}-\sum_{j \in \Lambda_{O}} u_{1, O, j} \\
& \Longleftrightarrow-2 \cdot \eta_{O}^{k}\left(\Lambda_{O} \backslash K\right)=\eta_{\mathrm{sym}} \\
& \Longleftrightarrow-2 \cdot \sum_{j \in \Lambda_{O}} u_{1, O, j}=\sum_{j \in \Lambda_{O}} u_{1, O, j}-\eta_{O}^{k}\left(\Lambda_{O}\right) \\
& \Longleftrightarrow-u_{0, O}-\frac{\sum_{j \in \Lambda_{O}} u_{1, O, j}}{2}-\frac{\eta_{O}^{k}\left(\Lambda_{O}\right)}{2}=0 \\
& \Longleftrightarrow u_{0, O}+\frac{\sum_{j \in \Lambda_{O}} u_{1, O, j}}{2}+\frac{\eta_{O}^{k}\left(\Lambda_{O}\right)}{2}=0
\end{aligned}
$$

which is the expected result.

Now, let us show that the symmetric property of the nonlinear interaction potential can be expressed through conditional probabilities $\Phi_{O}$ 's.

Lemma 3. Let $N$ be an arbitrary SNTBAN of order $k$. Then, the following equation holds:

$$
\begin{aligned}
\forall K \subseteq \Lambda_{O}: u_{0, O}+\frac{\sum_{j \in \Lambda_{O}} u_{1, O, j}}{2}+\frac{\eta_{O}^{k}\left(\Lambda_{O}\right)}{2}=0 \Longleftrightarrow \\
\Phi_{O}\left(K, \Lambda_{O} \backslash K\right)+\Phi_{O}\left(\Lambda_{O} \backslash K, K\right)=1 .
\end{aligned}
$$

Proof. Let us consider Equation 5. In order for its left member to hold, let us remark that the nonlinear term of $N \eta_{O}^{k}\left(\Lambda_{O}\right)$ needs to equal $-2 \cdot u_{0, O}-$ $\sum_{j \in \Lambda_{O}} u_{1, O, j}$. Now, considering $\eta_{O}^{k}\left(\Lambda_{O}\right)=-2 \cdot u_{0, O}-\sum_{j \in \Lambda_{O}} u_{1, O, j}$ as an hypothesis, let us show that the right member of Equation 5 holds for every subset $K$ of the strict neighbourhood of the central automaton $O$. To do so, without loss of generality, let us consider an arbitrary set $K$ such that $K \subseteq \Lambda_{O}$. Then, it suffices to multiply $\Phi_{O}\left(K, \Lambda_{O} \backslash K\right)$ by:

$$
1=\frac{e^{-2 \cdot u_{0, O}-\sum_{j \in \Lambda_{O}} u_{1, O, j}-\eta_{O}^{k}\left(\Lambda_{O}\right)}}{e^{-2 \cdot u_{0, O}-\sum_{j \in \Lambda_{O}} u_{1, O, j}-\eta_{O}^{k}\left(\Lambda_{O}\right)}} .
$$

Thus, we obtain:

$$
\begin{aligned}
\Phi_{O}\left(K, \Lambda_{O} \backslash K\right)=\frac{e^{u_{0, O}+\sum_{j \in K} u_{1, O, j}+\eta_{O}^{k}(K)}}{1+e^{u_{0, O}+\sum_{j \in K} u_{1, O, j}+\eta_{O}^{k}(K)}} \times \\
\frac{e^{-2 \cdot u_{0, O}-\sum_{j \in \Lambda_{O}} u_{1, O, j}-\eta_{O}^{k}\left(\Lambda_{O}\right)}}{e^{-2 \cdot u_{0}, O-\sum_{j \in \Lambda_{O}} u_{1, O, j}-\eta_{O}^{k}\left(\Lambda_{O}\right)}} .
\end{aligned}
$$


Let us denote by $\delta$ the result of the multiplication of the denominators of the fractions in the previous equation. We have:

$$
\delta=e^{-2 \cdot u_{0, O}-\sum_{j \in \Lambda_{O}} u_{1, O, j}-\eta_{O}^{k}\left(\Lambda_{O}\right)}+e^{-u_{0, O}-\sum_{j \in \Lambda_{O} \backslash K} u_{1, O, j}+\eta_{O}^{k}(K)-\eta_{O}^{k}\left(\Lambda_{O}\right)} .
$$

That leads us to simplify Equation 6 such that:

$$
\Phi\left(K, \Lambda_{O} \backslash K\right)=\frac{e^{-u_{0, O}-\sum_{j \in \Lambda_{O} \backslash K} u_{1, O, j}+\eta_{O}^{k}(K)-\eta_{O}^{k}\left(\Lambda_{O}\right)}}{\delta} .
$$

By hypothesis on the nonlinear interaction potential of $N$, we know that nonlinear term $\eta_{O}^{k}\left(\Lambda_{O}\right)=-2 \cdot u_{0, O}-\sum_{j \in \Lambda_{O}} u_{1, O, j}$. As a consequence, we have:

$$
e^{-2 \cdot u_{0, O}-\sum_{j \in \Lambda_{O}} u_{1, O, j}-\eta_{O}^{k}\left(\Lambda_{O}\right)}=e^{0}=1 .
$$

Moreover, given that nonlinear term $\eta_{O}^{k}$ is symmetric, we can write that:

$$
\begin{aligned}
\Phi\left(K, \Lambda_{O} \backslash K\right) & =\frac{e^{-u_{0, O}-\sum_{j \in \Lambda_{O} \backslash K} u_{1, O, j}-\eta_{O}^{k}\left(\Lambda_{O} \backslash K\right)}}{1+e^{-u_{0, O}-\sum_{j \in \Lambda_{O} \backslash K} u_{1, O, j}-\eta_{O}^{k}\left(\Lambda_{O} \backslash K\right)}} \\
& =1-\frac{e^{u_{0, O}+\sum_{j \in \Lambda_{O} \backslash K} u_{1, O, j}+\eta_{O}^{k}\left(\Lambda_{O} \backslash K\right)}}{1+e^{u_{0, O}+\sum_{j \in \Lambda_{O} \backslash K} u_{1, O, j}+\eta_{O}^{k}\left(\Lambda_{O} \backslash K\right)}} \\
& =1-\Phi\left(\Lambda_{O} \backslash K, K\right) .
\end{aligned}
$$

As a result, the right member of Equation 5 holds:

$$
\Phi\left(\Lambda_{O} \backslash K, K\right)=1-\Phi\left(K, \Lambda_{O} \backslash K\right) .
$$

Now, expanding left and right members of the equation above leads to:

$$
\frac{e^{u_{0, O}+\sum_{j \in \Lambda_{O} \backslash K} u_{1, O, j}+\eta_{O}^{k}\left(\Lambda_{O} \backslash K\right)}}{1+e^{u_{0, O}+\sum_{j \in \Lambda_{O} \backslash K} u_{1, O, j}+\eta_{O}^{k}\left(\Lambda_{O} \backslash K\right)}}=1-\frac{e^{u_{0, O}+\sum_{j \in K} u_{1, O, j}+\eta_{O}^{k}(K)}}{1+e^{u_{0, O}+\sum_{j \in K} u_{1, O, j}+\eta_{O}^{k}(K)}},
$$

which is equivalent to:

$$
\frac{e^{u_{0, O}+\sum_{j \in \Lambda_{O} \backslash K} u_{1, O, j}+\eta_{O}^{k}\left(\Lambda_{O} \backslash K\right)}}{1+e^{u_{0, O}+\sum_{j \in \Lambda_{O} \backslash K} u_{1, O, j}+\eta_{O}^{k}\left(\Lambda_{O} \backslash K\right)}}=\frac{e^{-u_{0, O}-\sum_{j \in K} u_{1, O, j}-\eta_{O}^{k}(K)}}{1+e^{-u_{0, O}-\sum_{j \in K} u_{1, O, j}-\eta_{O}^{k}(K)}} .
$$

Let us proceed to the following changes of variables: let $\delta_{1}$ (resp. $\delta_{2}$ ) be the denominator of the left member (resp. of the right member) and $\eta_{1}$ (resp. $\eta_{2}$ ) the numerator of the left member (resp. of the right member) of Equation 7 above. We have then:

$$
\begin{aligned}
\frac{\eta_{1}}{\delta_{1}}=\frac{\eta_{2}}{\delta_{2}} & \Longleftrightarrow \frac{\eta_{1} \cdot \delta_{2}}{\delta_{1} \cdot \delta_{2}}=\frac{\eta_{2} \cdot \delta_{1}}{\delta_{2} \cdot \delta_{1}} \\
& \Longleftrightarrow \eta_{1} \cdot \delta_{2}=\eta_{2} \cdot \delta_{1} .
\end{aligned}
$$


Let $\psi$ be such that:

$$
\psi=e^{\sum_{j \in \Lambda_{O} \backslash K} u_{1, O, j}-\sum_{j \in K} u_{1, O, j}+\eta_{O}^{k}\left(\Lambda_{O} \backslash K\right)-\eta_{O}^{k}(K)} .
$$

As a consequence, we derive that:

$$
\begin{aligned}
\frac{\eta_{1}}{\delta_{1}}=\frac{\eta_{2}}{\delta_{2}} & \Longleftrightarrow \eta_{1}+\psi=\eta_{2}+\psi \\
& \Longleftrightarrow \eta_{1}=\eta_{2},
\end{aligned}
$$

and then:

$$
\begin{aligned}
& \frac{\eta_{1}}{\delta_{1}}=\frac{\eta_{2}}{\delta_{2}} \Longleftrightarrow e^{u_{0, O}+\sum_{j \in \Lambda_{O} \backslash K} u_{1, O, j}+\eta_{O}^{k}\left(\Lambda_{O} \backslash K\right)}=e^{-u_{0, O}-\sum_{j \in K} u_{1, O, j}-\eta_{O}^{k}(K)} \\
& \Longleftrightarrow u_{0, O}+\sum_{j \in \Lambda_{O} \backslash K} u_{1, O, j}+\eta_{O}^{k}\left(\Lambda_{O} \backslash K\right)= \\
& \quad-u_{0, O}-\sum_{j \in K} u_{1, O, j}-\eta_{O}^{k}(K) \\
& \Longleftrightarrow \eta_{O}^{k}(K)=-2 \cdot u_{0, O}-\sum_{j \in \Lambda_{O} \backslash K} u_{1, O, j}- \\
& \sum_{j \in K} u_{1, O, j}-\eta_{O}^{k}\left(\Lambda_{O} \backslash K\right) .
\end{aligned}
$$

Thus, we have:

$$
\frac{\eta_{1}}{\delta_{1}}=\frac{\eta_{2}}{\delta_{2}} \Longleftrightarrow \eta_{O}^{k}(K)=-2 \cdot u_{0, O}-\sum_{j \in \Lambda_{O}} u_{1, O, j}-\eta_{O}^{k}\left(\Lambda_{O} \backslash K\right) .
$$

Hence, by hypothesis, we write:

$$
\frac{\eta_{1}}{\delta_{1}}=\frac{\eta_{2}}{\delta_{2}} \Longleftrightarrow \eta_{O}^{k}(K)=\eta_{O}^{k}\left(\Lambda_{O}\right)-\eta_{O}^{k}\left(\Lambda_{O} \backslash K\right) .
$$

From Lemma 2 and since the reasoning has been done for an arbitrary $K$, previous equation can be generalised for every $K \subseteq \Lambda_{O}$ and rewritten as:

$$
\begin{aligned}
\forall K \subseteq \Lambda_{O}: \Phi_{O}\left(K, \Lambda_{O} \backslash K\right)+\Phi_{O} & \left(\Lambda_{O} \backslash K, K\right)=1 \\
& \Longleftrightarrow u_{0, O}+\sum_{j \in \Lambda_{O}} \frac{u_{1, O, j}}{2}+\frac{\eta_{O}^{k}\left(\Lambda_{O}\right)}{2}=0,
\end{aligned}
$$

which is the expected result.

From Lemmas 1, 2 and 3, it is easy to derive the following theorem that highlights a sufficient condition of phase transitions in SNTBANs of order $k$ on $\mathbb{Z}^{2}$.

TheOREM. Given $N$ a SNTBAN of order $k$, then the following equation holds:

$$
\eta_{O}^{k}(K)=\eta_{O}^{k}\left(\Lambda_{O}\right)-\eta_{O}^{k}\left(\Lambda_{O} \backslash K\right) \Longrightarrow \operatorname{Det} \mathcal{M}=0,
$$

which means that the symmetry property of the non linear term is a sufficient condition for Det $\mathcal{M}$ to vanish, allowing consequently phase transitions to occur. 

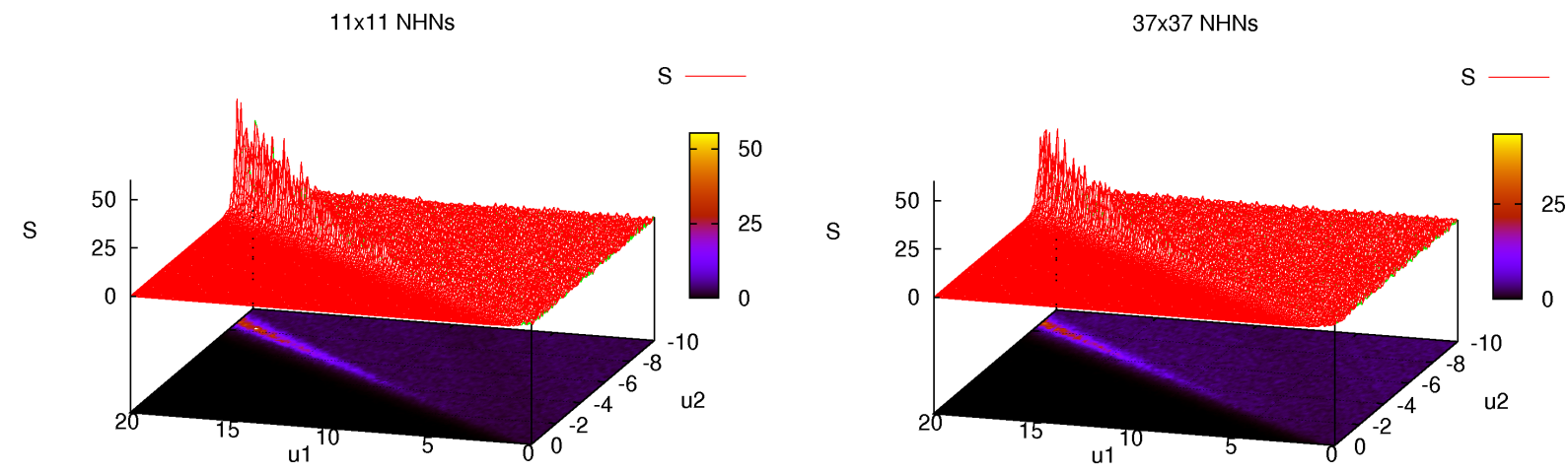

$131 \times 131$ NHNs

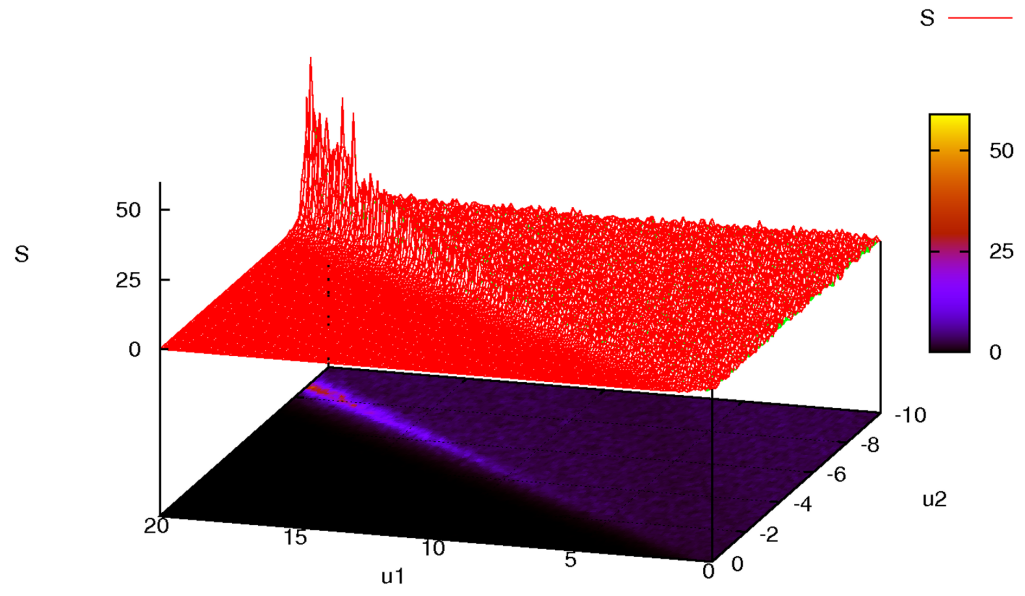

Fig. 4. Phase transitions emerging in the neighbourhood of the equation $\mathbf{u}_{\mathbf{0}}+\mathbf{2} \cdot \mathbf{u}_{\mathbf{1}}+\mathbf{5} \cdot \mathbf{u}_{\mathbf{2}}=\mathbf{0}$ for isotropic and translation invariant attractive SNTBANs of order $\mathbf{3}$ of increasing sizes: (Top left) $\mathbf{1 1} \times \mathbf{1 1}$, (Top right) $\mathbf{3 7} \times \mathbf{3 7}$ and (Bottom) $131 \times 131$.

Proof. From Lemma 1 and because of the parity of the cardinal of $\Lambda_{O}$ (the equivalent number of subsets of $\Lambda_{O}$ of even cardinal equals that of subsets of $\Lambda_{O}$ of odd cardinal), we can write:

$$
\begin{aligned}
& \operatorname{Det} \mathcal{M}=0 \Longleftrightarrow \sum_{K \subseteq \Lambda_{O}}(-1)^{\left|\Lambda_{O} \backslash K\right|} \cdot \Phi_{O}\left(K, \Lambda_{O} \backslash K\right)=0 \\
& \Longleftrightarrow \sum_{K \subseteq \Lambda_{O}}(-1)^{\left|\Lambda_{O} \backslash K\right|} \times \\
& \frac{1}{2} \cdot\left(\Phi_{O}\left(K, \Lambda_{O} \backslash K\right)+\Phi_{O}\left(\Lambda_{O} \backslash K, K\right)\right)=0 .
\end{aligned}
$$


Then, from Lemma 3, we have:

$$
\operatorname{Det} \mathcal{M}=0 \Longleftrightarrow \sum_{K \subseteq \Lambda_{O}}(-1)^{\left|\Lambda_{O} \backslash K\right|} \cdot \frac{1}{2}=0,
$$

which is always true with respect to the general hypothesis of symmetry of the nonlinear term (see Equation 5). As a result, from Lemmas 1, 2 and 3, we obtain:

$$
u_{0, O}+\frac{\sum_{j \in \Lambda_{O}} u_{1, O, j}}{2}+\frac{\eta_{O}^{k}\left(\Lambda_{O}\right)}{2}=0 \Longrightarrow \operatorname{Det} \mathcal{M}=0
$$

which is the expected result.

From this result, we obtain the expected sufficient condition under which the determinants of the projectivity matrices of such stochastic systems are null. As a consequence, we will now show by simulations that such a condition represents an empirical necessary condition of phase transitions emergence in the dynamics of SNTBANs of any order $k$ on $\mathbb{Z}^{2}$. Let us insist on the fact that what we propose here constitutes a generalisation to the nonlinear case of the classical condition of phase transitions given in [22] for attractive linear Ising model. Indeed, whatever the order of nonlinearity is, as it is presented in Equation 8, it suffices to add to Ruelle's classical equation $\nu+2 \cdot \varpi=0$ (where $\nu$ and $\varpi$ denote respectively the singleton and couple potentials) the half of the value of the symmetric nonlinear term to obtain the empirical equation of phase transitions. In other terms, the environment modelled by boundary conditions has a specific influence on the dynamical behaviours of SNTBANs on $\mathbb{Z}^{2}$ when the latter are characterised by a nonlinear potential equal to the opposite of $\varsigma$ (where $\varsigma$ represents the sum of twice the singleton potential and of the sum of the couple potentials).

\subsection{Simulation results}

Consider in the sequel the following notation simplifications: $u_{0}=u_{0, O}, u_{1}=$ $u_{1, O, j}$ and $u_{2}=u_{2, O,\langle\ell, m\rangle}$, with $j \in \Lambda_{O}$ and $\ell, m \in \mathcal{N}_{O}$. In order to support the theoretical results presented above and illustrate Equation 8, we performed simulations measuring the differences between central activities of SNTBANs of order 3 subjected to boundary conditions where $\partial_{\text {ext }}^{0} N$ is defined as $\forall t \in \mathcal{T}, \forall i \in$ $\partial_{\text {ext }} N: x_{i}(t)=0$ and $\partial_{\text {ext }}^{1} N$ is defined as $\forall t \in \mathcal{T}, \forall i \in \partial_{\text {ext }} N: x_{i}(t)=1$, as proposed in Section 3. The choice of these two types of boundary conditions, called "extremal boundary conditions", comes from results of $[26,36]$ showing that although any kind of boundary condition has a significant influence on linear TBANs, extremal ones are the most impacting in the case of attractive networks. Let us add that in these simulations, the transient time equals 10000 time steps and the sampling time equals 1000 time steps. Each panel of Figure 4 pictures the average dissimilarity measures obtained from 5 computations of asymptotic dynamical behaviours of 20000 SNTBANs of order 3 of respective sizes $11 \times 11,37 \times 37$ and $131 \times 131$ (every couple of values on the plane $\left(0, u_{1}, u_{2}\right)$ corresponds to a SNTBAN characterised by parameters $u_{0}=0,0 \leq u_{1} \leq 20$ 
and $-10 \leq u_{2} \leq 0$ with a step variation of 0.1 ). Sizes chosen allow to obtain results on networks of three different orders of magnitude. Thereby, simulation results give pertinent observations of the theoretical results discussed above. In particular, all panels reveal that measures are significantly strictly positive on a phase domain located in the neighbourhood of the equation $u_{0}+2 \cdot u_{1}+5 \cdot u_{2}=$ 0 . As the networks considered in these simulations are nonlinear networks of order 3, the nonlinear term that needs to be taken into account according to the theoretical results of Section 4.2 is computed as the sum of all possible triplet potentials. More precisely, in this case, since triplet potentials on $O$ are computed depending on its neighbourhood (see Equation 1), $O$ itself needs to be taken into account for the composition of couples that possibly impact its state over time, as pictured in Figure 3. In the context of this example, we have $\frac{\eta_{O}^{k}\left(\Lambda_{O}\right)}{2}=\sum_{\substack{j_{1}, j_{2} \in \mathcal{N}_{i} \\ j_{1} \neq j_{2}}} u_{2, i,\left\langle j_{1}, j_{2}\right\rangle} \cdot x_{j_{1}}(t) \cdot x_{j_{2}}(t)=\left(\begin{array}{l}5 \\ 2\end{array}\right) \cdot \frac{u_{2}}{2}=5 \cdot u_{2}$. Hence, for SNTBANs of order 3 , Equation 8 is $u_{0}+2 \cdot u_{1}+5 \cdot u_{2}=0 \Longrightarrow \operatorname{Det} \mathcal{M}=0$, which is the expected result.

Amongst the important results obtained from these simulations, note that they allow to precise the parameters values of networks subjected to the influence of boundary conditions. Indeed, what is remarkable in Figure 3 is that, in the neighbourhood of the origin of the diagram where $u_{1} \approx u_{2} \approx 0$, the invariant measure is unique (the dissimilarity measure is almost null). This specific neighbourhood that induces ergodicity of the underlying Markov chains is for now only visible by simulations. Furthermore, these simulations show that the phase transition domain obtained from theory is unique, which implies that the symmetry of the nonlinear term is also a necessary condition for Det $\mathcal{M}=0$ to hold. Eventually, we can conclude that attractive SNTBANs are globally robust against their environment. Indeed, as pictured in Figure 4, the major part of the plane $\left(O, u_{1}, u_{2}\right)$ admits a dissimilarity measure $S$ close to 0 . Nevertheless, one specific family of networks, characterised by specific structural parameters satisfying Equation 8, is subjected to structural instabilities due to boundary conditions fluctuations, which induces a high power of the environment. This empirical result allows to conclude that the symmetry property of the nonlinear term of local transition function of SNTBANs on $\mathbb{Z}^{2}$ is a necessary condition for phase transition to emerge and, hence, for SNTBANs to be significantly dependent on their environment.

\section{Conclusion and discussion}

In this work has been presented a computer-assisted approach (due to the real difficulty to exhibit a formal characterisation of domains of phase transitions) which led us to highlight the singular power of the environment on the dynamical behaviours of attractive SNTBANs dived into $\mathbb{Z}^{2}$. Besides the improvements of the theoretical method to get an explicit formal characterisation of phase transitions on which we will not dwell, this work opens several interesting perspectives.

First, the concept of nonlinearity could be used to model protein complexes functionally rather than structurally in the context of genetic regulation net- 
works. Indeed, nonlinearity constitutes a way to integrate protein complexes into the local transition functions. At present, protein complexes are represented by adding specific nodes into the underlying interaction graphs of networks. This implies a significant increase of the sizes of inputs from the algorithmic point of view. Thus, nonlinearity could constitute a significant gain in many algorithmic tools aiming at simulating the dynamical behaviours of such networks modelled by SNTBANs. It also would be relevant to study how could nonlinearity be introduced in other models of genetic regulation networks, such as Thomas' networks [10] for instance.

As explained in the introduction, although the results presented cannot be directly applied to real biological networks because of the purely theoretical nature of networks considered, they support the insight that real networks should not be studied without considering the influence of their environment. Indeed, generalising the definition of a network boundary to the set of sources of its underlying interaction graph (in terms of digraphs) in the context of real networks, genetic regulation networks, for instance, are networks subjected to the specific action of micro-RNAs (whose post-transcriptional classical effect is to inhibit the translation of the messenger-RNAs into proteins) and hormones (whose flows cross the cells lipidic membranes and result in specific interactions with genes of regulation networks) that can be considered as boundaries. In this context, we have shown in previous works that the approach detailed here can be used to understand, explain and predict biological phenomena. For instance, in [37], we have proposed a formalised explanation of the influence of the hormone Gibberellin on the floral morphogenesis of Arabidopsis. Other applications to Systems Biology of this approach can be found in [36]. Now, other studies on the influence of micro-RNAs $[38,39]$ on real genetic networks need to be performed to make our method finer and precise more its application field. In this context, studying the cell cycle and regulation networks involved in the control of the immune system seems to be a good starting point $[40,41]$.

Eventually, let us conclude on more theoretical perspectives. One the one hand, it would be interesting to model a dynamical environment. In this work, the environment is represented by static boundary conditions which can be encoded as vectors. To develop the method, the idea would be to represent the environment by boundary conditions encoded as matrices representing for instance a periodic change of the environment. Studies on the nature of the boundary conditions the most powerful for breaking robustness with respect to boundaries, in relation to the behaviours of networks, would be relevant. On the other hand, in order to keep the theoretical context but make a larger step towards more realistic biological networks, we would like to study the behaviours of less constrained SNTBANs. Beyond the isotropic and translation invariant constraints, it would be relevant to relax topological constraints and study for instance the impact of boundary conditions on SNTBANs subjected to stochastic perturbations which would aim at removing randomly proportions of arcs in underlying interaction graphs. 
Acknowledgements This work has been partially supported by the ANR project Synbiotic, the RNSC project Météding and the IXXI project Maajes.

\section{References}

1. McCulloch, W.S., Pitts, W.: A logical calculus of the ideas immanent in nervous activity. Bull. Math. Biophys. 5 (1943) 115-133

2. Goles, E., Olivos, J.: The convergence of symmetric threshold automata. Inform. Control 51 (1981) 98-104

3. Goles-Chacc, E., Fogelman-Soulie, F., Pellegrin, D.: Decreasing energy functions as a tool for studying threshold networks. Discrete Appl. Math. 12 (1985) 261-277

4. Goles, E., Vichniac, G.Y.: Lyapunov functions for parallel neural networks. AIP Conference Proceedings 151 (1986) 165-181

5. Hopfield, J.J.: Neural networks and physical systems with emergent collective computational abilities. Proc. Natl. Acad. Sci. U. S. A. 79 (1982) 2554-2558

6. Hopfield, J.J.: Neurons with graded response have collective computational properties like those of two-state neurons. Proc. Natl. Acad. Sci. U. S. A. 81 (1984) 3088-3092

7. Hopfield, J.J., Tank, D.W.: "Neural" computation of decisions in optimization problems. Biol. Cybern. 52 (1985) 141-152

8. Kauffman, S.A.: Metabolic stability and epigenesis in randomly constructed genetic nets. J. Theor. Biol. 22 (1969) 437-467

9. Glass, L., Kauffman, S.A.: Co-operative components, spatial localization and oscillatory cellular dynamics. J. Theor. Biol. 34 (1972) 219-237

10. Thomas, R.: Boolean formalization of genetic control circuits. J. Theor. Biol. 42 (1973) 563-585

11. Thomas, R.: On the relation between the logical structure of systems and their ability to generate multiple steady states or sustained oscillations. Springer Series in Synergetics 9 (1981) 180-193

12. Mendoza, L., Alvarez-Buylla, E.: Dynamics of the genetic regulatory network for Arabidopsis thaliana flower morphogenesis. J. Theor. Biol. 193 (1998) 307-319

13. Aracena, J., Ben Lamine, S., Mermet, O., Cohen, O., Demongeot, J.: Mathematical modeling in genetic networks: relationships between the genetic expression and both chromosomic breakage and positive circuits. IEEE Trans. Syst. Man Cybern. B 33 (2003) 825-834

14. Thom, R.: Structural Stability and Morphogenesis. Benjamin (1976)

15. Seth, A.K., Edelman, G.M.: Environment and behavior influence the complexity of evolved neural networks. Adapt. Behav. 12 (2004) 5-20

16. Wang, Z., Shu, H., Fang, J., Liu, X.: Robust stability for stochastic Hopfield neural networks with time delays. Nonlinear Anal. - Real 7 (2006) 1119-1128

17. Wagner, A.: Robustness against mutations in genetic networks of yeast. Nature Genet. 24 (2000) 355-361

18. Kitano, H.: Biological robustness. Nat. Rev. Genet. 5 (2004) 826-837

19. Ising, E.: Beitrag zür theorie des ferromagnetismus. Zeitschrift für Physics 31 (1925) 253-258

20. Dobrushin, R.L.: Gibbsian random fields for lattice systems with pairwise interactions. Funct. Anal. Appl. 2 (1968) 292-301

21. Dobrushin, R.L.: The problem of uniqueness of a Gibbsian random field and the problem of phase transitions. Funct. Anal. Appl. 2 (1968) 302-312 
22. Ruelle, D.: Statistical Mechanics: Rigourous Results. W. A. Benjamin (1969)

23. Wu, X.N., Wu, F.Y.: Exact results for lattice models with pair and triplet interactions. J. Phys. A 22 (1989) L1031

24. Wu, F.Y.: Rigorous results on the triangular Ising model with pair and triplet interactions. Phys. Lett. A 153 (1991) 73-75

25. Qin, Y., Yang, Z.R.: Critical dynamics of the kinetic Ising model with triplet interaction on Sierpinski-gasket-type fractals. Phys. Rev. B 46(18) (1992) 1128411289

26. Demongeot, J., Jézéquel, C., Sené, S.: Boundary conditions and phase transitions in neural networks. Theoretical results. Neural Netw. 21 (2008) 971-979

27. Demongeot, J., Sené, S.: Boundary conditions and phase transitions in neural networks. Simulation results. Neural Netw. 21 (2008) 962-970

28. Sené, S.: Influence des conditions de bord dans les réseaux d'automates booléens à seuil et application à la biologie. PhD thesis, Université Joseph Fourier - Grenoble $1(2008)$

29. Goles, E., Martínez, S.: Neural and Automata Networks. Volume 58 of Mathematics and Its Application. Kluwer Academic Publisher (1990)

30. Robert, F.: Iterations sur des ensembles finis et automates cellulaires contractants. Linear Alg. Appl. 29 (1980) 393-412

31. Robert, F.: Discrete Iterations: a Metric Study. Volume 6 of Springer Series in Computational Mathematics. Springer (1986)

32. Martinelli, F.: On the two-dimensional dynamical Ising model in the phase coexistence region. J. Stat. Phys. 76 (1994) 1179-1246

33. Hinton, G.E., Sejnowski, T.J.: Optimal perceptual inference. In: Proceedings of CVPR'1983, IEEE Press (1983) 448-453

34. Ackley, D.H., Hinton, G.E., Sejnowski, T.J.: A learning algorithm for Boltzmann machines. Cognitive Sci. 9 (1985) 147-169

35. Demongeot, J.: Asymptotic inference for Markov random field on $\mathbb{Z}^{d}$. Springer Series in Synergetics 9 (1981) 254-267

36. Ben Amor, H., Demongeot, J., Sené, S.: Structural sensitivity of neural and genetic networks. In: Proceedings of MICAI'08. Volume 5317 of LNCS., Springer (2008) 973-986

37. Demongeot, J., Goles, E., Morvan, M., Noual, M., Sené, S.: Attraction basins as gauges of the robustness against boundary conditions in biological complex systems. PLoS One 5 (2010) e11793

38. Calin, G.A., Croce, C.M.: MicroRNA signatures in human cancers. Nat. Rev. Cancer 6 (2006) 857-866

39. Carleton, M., Cleary, M.A., Linsley, P.S.: MicroRNAs and cell cycle regulation. Cell Cycle 6 (2007) 2127-2132

40. Demongeot, J., Elena, A., Noual, M., Sené, S., Thuderoz, F.: "Immunetworks", intersecting circuits and dynamics. J. Theor. Biol. 280 (2011) 19-33

41. Demongeot, J., Noual, M., Sené, S.: Combinatorics of Boolean automata circuits dynamics. Discrete Appl. Math. (2011) Submitted. 